\title{
The Impact of Adding a Nitric Oxide Donor as Adjuvant Therapy to Clomiphene Citrate to Improve Pregnancy Rate in Polycystic Ovarian Syndrome : A Randomized Controlled Trial
}

Article

\author{
Yasmin A. Bassiouny and Ayman A. Hassan
}

Department of Obstetrics and Gynecology, Faculty of Medicine, Cairo University, Egypt

\begin{abstract}
Aim: This study aimed to compare the effect of adding a nitric oxide donor to clomiphene citrate in women with polycystic ovarian syndrome for induction of ovulation to attempt and reverse the anti-estrogenic effect and thus improve the clinical pregnancy rate.

Material and Methods: This is an open labelled randomized controlled trial in a University Hospital infertility outpatient clinic. Eighty participants, fulfilling the inclusion criteria were enrolled in the study from January 2019 till December 2019. They were divided into 2 groups, group A received clomiphene citrate (CC) and Isosorbide mononitrate (ISMN), while group B received CC only. The primary outcome was the clinical pregnancy rate.

Results: The clinical pregnancy rate was higher in the ISMN group but did not show statistical significance. Although, the addition of ISMN had a favorable outcome on the number of follicles, endometrial volume, cervical mucus score, uterine artery RI.

Conclusion: The addition of nitric oxide donors to $\mathrm{CC}$ in the induction of ovulation in polycystic ovarian syndrome improved cycle parameters but did not significantly increase clinical pregnancy rates and thus should be cautiously used for such purpose.
\end{abstract}

Received: 10 July 2020, Accepted: 14 September 2020

Key Words: Anovulation, induction of ovulation, isosorbide mononitrate, PCOS

Corresponding Author: Yasmin A. Bassiouny, Department of Obstetrics and Gynecology, Faculty of Medicine, Cairo University, Egypt, Tel.: 01001720751, E-mail: bassionyyasmin@gmail.com

ISSN: 2090-7265, August 2021, Vol.11, No. 3

\section{INTRODUCTION}

Polycystic ovary syndrome (PCOS) is one of the most common endocrinopathies affecting $5-10 \%$ of reproductive age women ${ }^{[1]}$. Ovarian hyperandrogenism and chronic anovulation are the main criteria of the disease ${ }^{[2]}$.

Clomiphene citrate $(\mathrm{CC})$ is still the first line medication for the induction of ovulation in PCOS women ${ }^{[3]}$ with reported ovulation rate of $49 \%$ and a pregnancy rate of $23.9 \%{ }^{[4]}$. The anti-estrogenic action of CC explains the difference between achieving ovulation and getting pregnant ${ }^{[5]}$.

Treatment of such cases is a challenging problem with either gonadotrophins or laparoscopic ovarian drilling. Both modalities are expensive and have remarkable risks ${ }^{\left[{ }^{[]}\right.}$.

In developing countries where patients have financial constraints, there is an enormous need for exploring a safe, well tolerated treatment that is less costly and could be an effective adjuvant for $\mathrm{CC}$ to overcome its antagonistic action.
Nitric Oxide (NO) donors are direct smooth muscle relaxant that promote vasodilatation and improve the blood supply of organs. Its metabolites are detected in many biological fluids including follicular fluid and endometrial fluid ${ }^{[7]}$. NO is important for follicular growth and ovulation, it also is necessary for decidualization, thus playing a vital role in implantation and participates in the acrosome reaction of spermatozoa during capacitation ${ }^{[8]}$ and increases cervical mucus secretion at ovulation ${ }^{[9]}$.

\section{AIM OF THE WORK}

The aim of this prospective study is to compare the effect of backing-up CC with a NO donor to reverse its anti-estrogenic effect during ovulation induction in PCOS and improve the clinical pregnancy rate.

\section{PATIENTS AND METHODS}

The study included 80 participants among those attending the fertility clinic in Kasr Al-Aini hospital, Cairo University, Egypt in the period from January 2019 to December 2019. 
The protocol was approved by the Research committee of the department of obstetrics and gynecology, Cairo University Hospital and all patients gave informed consent before inclusion in the trial.

Inclusion criteria : Age between 20-39 years. All patients diagnosed with PCOS according to the Rotterdam criteria, in which 2 of 3 criteria had to be present: oligo or amenorrhea, clinical or biochemical hyperandrogenemia or polycystic ovaries by vaginal ultrasound ${ }^{[10]}$. All patients had previously received CC $100 \mathrm{mg} /$ day for 5 days starting from day 2 with successful ovulation as documented by folliculometry and midluteal serum progesterone with no pregnancy achieved. All patients had a normal Hysterosalpingography. All partners had a normal semen analysis. All patients had normal serum prolactin and thyroid function.

Exclusion criteria : Any other cause of infertility different from anovulation. Diabetes or other endocrinological disorders. Age less than 20 or more than 39 years. Presence of ovarian cyst $>2 \mathrm{~cm}$ on the second day of the cycle. Patients who have received gonadotrophins in the preceding 6 months. Patients who have done unilateral or bilateral ovarian drilling. Allergy to NO donors.

Patients were randomized according to a computer generated random numeric table prepared by an independent statistician to be allocated to one of 2 groups: group A received CC $100 \mathrm{mg} /$ day (Clomid, Sanofi) from day 2 until day 6 of the cycle and Isosorbide mononitrate (ISMN) $20 \mathrm{mg}$ tablet (EFFOX, Minapharm, Shwartz pharma) applied vaginally from day 1 to day 12 of the cycle. While group B received only $\mathrm{CC}$ in the same manner as group A. There was no blinding to either the participants or study conductors. Patients were not denied their medical care if they choose not to participate or leave the study at any time.

For all patients, cycles were monitored by transvaginal sonography for the mean follicular diameter on day 10,12 and 14 of the cycle and endometrial volume was calculated by VOCAL program using the Voluson 730 Pro ultrasound machine (GE, NYSE, USA). When one leading follicle reached $18 \mathrm{~mm}$ in diameter, cervical mucus score was recorded ${ }^{[11]}$ and uterine artery RI was measured.

Ovulation triggering was done by giving $10000 \mathrm{IU}$ of HCG (Choriomon, IBSA). Patients were advised to have intercourse 36 hours after the HCG injection. Serum progesterone was measured 1 week after follicle rupture on day 21 . Serum B-HCG was done 14 days after HCG injection.

The primary outcome measure was the occurrence of clinical pregnancy which is defined as detecting fetal pulsations after 5 weeks from the pregnancy test.
The secondary outcome measures were the number of follicles $>18 \mathrm{~mm}$, endometrial volume, cervical mucus score, uterine artery RI and serum progesterone level.

\section{STATISTICAL ANALYSIS:}

\section{Statistical analysis}

Data were statistically described in terms of mean \pm standard deviation $( \pm \mathrm{SD})$, median and range, or frequencies (number of cases) and percentages when appropriate. Comparison between groups was performed using Student t-test for quantitative variables and Chi square $\left(\mathrm{c}^{2}\right)$ test for qualitative ones. $P$ values less than 0.05 was considered statistically significant. All statistical calculations were done using computer program SPSS (Statistical Package for the Social Science; SPSS Inc., Chicago, IL, USA) version 15 for Microsoft Windows.

Sample size estimation was calculated based on the primary outcome. Pregnancy rate after clomid induction in a setting of timed intercourse was estimated to be $22.2 \%$ per patient. Assuming a 20\% difference between groups, it was estimated that 30 women were required in each group of the study to detect a true difference at the $95 \%$ confidence interval with $80 \%$ power. We added 10 participants to each arm to compensate for subjects not attending follow up visits.

\section{RESULTS}

The flow chart for recruited patients is shown in (Figure 1). One hundred and twenty-seven participants were assessed for inclusion, 15 did not meet the inclusion criteria and 12 refused to participate in the study. Failure to reach a dominant follicle occurred in 2 patients in the ISMN group and in 1 patient in the $\mathrm{CC}$ group.

There was no statistically significant difference between the 2 groups regarding age, duration of infertility, BMI, clinical manifestations, and hormonal profile (Table 1).

All cycle parameters and outcomes are shown in (Table 2). There was a statistically higher number of mature follicles and shorter days of induction, in the ISMN group than the $\mathrm{CC}$ group.

The endometrial volume was statistically higher, and the uterine artery RI was less in the ISMN group. As well as the cervical mucus score at the time of ovulation which showed a statistically significant difference between the 2 groups being better in the ISMN group than the CC group.

Serum progesterone level on day 21 and clinical pregnancy rate did not show a statistically significant difference between the 2 groups. 


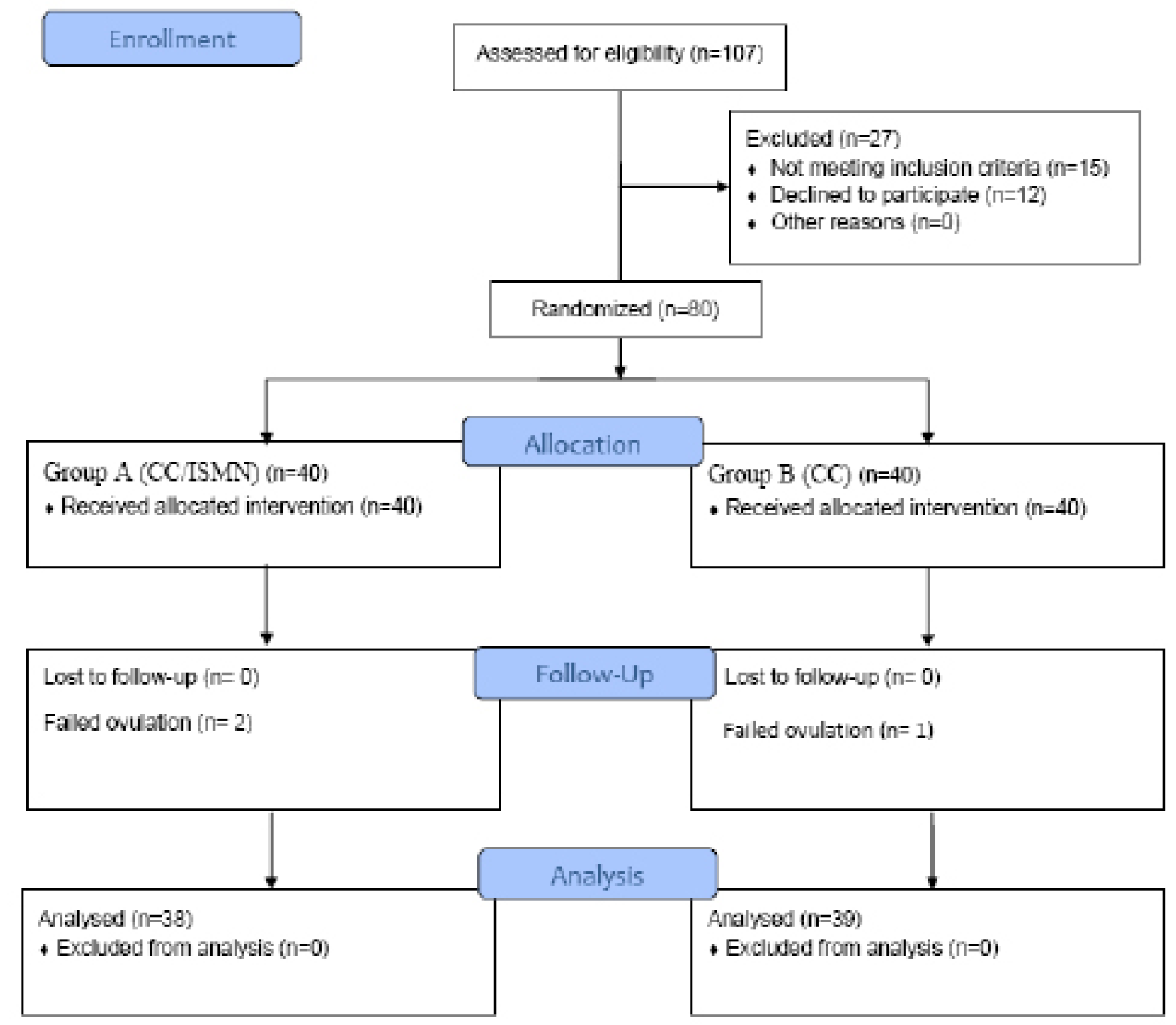

Fig. 1: Patient recruitment flow chart 
Table 1: Basal characteristics of patients

\begin{tabular}{|c|c|c|c|}
\hline Variables & Group A $(\mathrm{CC}+\mathrm{ISMN}) \mathrm{n}=38$ & Group B (CC only) $n=39$ & $P$-value \\
\hline Age & $29.4 \pm 4.7$ & $28.6 \pm 5.7$ & 0.849 \\
\hline Duration of infertility & $4.9 \pm 3.2$ & $4.6 \pm 3.4$ & 0.785 \\
\hline BMI & $30.9 \pm 3.6$ & $30.8 \pm 3.8$ & 0.740 \\
\hline Oligomenorrhea (n/n, \%) & $13 / 38,34.2 \%$ & $17 / 39,43.6 \%$ & 0.398 \\
\hline Amenorrhea $(\mathrm{n} / \mathrm{n}, \%)$ & $6 / 38,15.7 \%$ & $5 / 39,12.8 \%$ & 0.709 \\
\hline FSH & $7.2 \pm 1.9$ & $6 \pm 2.5$ & 0.057 \\
\hline LH & $9 \pm 2$ & $9.3 \pm 2.4$ & 0.141 \\
\hline Androgen & $0.68 \pm 0.26$ & $0.66 \pm 0.32$ & 0.720 \\
\hline
\end{tabular}

*P value $<0.05$ is considered statistically significant, all values presented as mean and standard deviation or percentage

Table 2: Cycle parameters and outcomes in the 2 groups

\begin{tabular}{|c|c|c|c|}
\hline Variables & Group A $(\mathrm{CC}+\mathrm{ISMN}) \mathrm{n}=38$ & Group B (CC only) $n=39$ & P-value \\
\hline Number of follicles & $1.5 \pm 0.6$ & $1.2 \pm 0.4$ & $<0.001^{*}$ \\
\hline Induction duration (days) & $12 \pm 0.9$ & $13.3 \pm 1$ & $<0.001^{*}$ \\
\hline Endometrial volume (ml) & $2.8 \pm 0.2$ & $2.2 \pm 0.1$ & $<0.001 *$ \\
\hline Uterine artery RI & $0.8 \pm 0.04$ & $0.89 \pm 0.03$ & $<0.001 *$ \\
\hline Cervical mucus score & $9.6 \pm 1.5$ & $9.3 \pm 1.9$ & $0.002 *$ \\
\hline Serum progesterone $(\mathrm{pg} / \mathrm{ml})$ & $9.7 \pm 1.5$ & $9.2 \pm 1.1$ & 0.147 \\
\hline Clinical Pregnancy rate & $7 / 38,18.4 \%$ & $5 / 39,12.8 \%$ & 0.498 \\
\hline
\end{tabular}

*P value $<0.05$ is considered statistically significant, all values presented as mean and standard deviation or percentage

\section{DISCUSSION:}

The enormous enthusiasm to explore different adjuvant therapy to $\mathrm{CC}$ induced cycles has risen from the challenge to overcome the disparity existing between the ovulation and pregnancy associated with CC therapy.

In the current study, the effect of adding a NO donor, namely ISMN, applied vaginally on the outcome of ovulation and pregnancy in PCOS women was assessed.
Showing improved follicular number, shortening the days of induction, improved endometrial volume and cervical mucous. The clinical pregnancy rate was higher but did not reach statistical significance. NO donors improve the ovarian blood supply which helps follicular maturation and selection, as they inhibit vascular smooth muscle contraction and promote vasodilatation ${ }^{[12]}$.

It is assumed that in PCOS, follicular development, maturation, ovulation, and implantation are impaired due 
to hormonal imbalance and vascular impairment ${ }^{[13]}$. On these basis, NO donors through vasodilatation increase the permeability of follicular epithelium to plasma proteins: mainly circulating FSH and LH, both hormones promote an increased production of IGF-1 which plays a critical role in follicular maturation and differentiation of granulose cells ${ }^{[14]}$.

Several studies investigated the effect of $\mathrm{NO}$ on the uterine blood flow, N-mono-methylarginine (a specific inhibitor of NO) was found to reduce uterine artery blood

flow by $60 \%{ }^{[15]}$ and in another study the vaginal administration of ISMN in women with recurrent miscarriage increased uterine artery and sub-endometrial blood flow ${ }^{[16]}$

ISMN is a smooth muscle relaxant that inhibits vasoconstriction and improve the blood supply of the endometrium promoting its growth and proliferation which agrees with Beny and Razik they also showed improvement in the endometrial thickness, resulting in higher pregnancy rate with vaginal ISMN and CC in their recruited participant ${ }^{\mathrm{s}[17]}$.

In a study that assessed the side effects of ISMN during the course of induction they found significantly more headache, flushing and palpitation. But they concluded that adding ISMN significantly improved the pregnancy rate $^{[18]}$.

Another study recruited patients with unexplained infertility also concluded significantly higher pregnancy rates ${ }^{[19]}$. In our study we reported higher pregnancy rates that did not reach statistical significance, this was the same conclusion that Ajam et al. reached in their study ${ }^{[20]}$.

The limitations of our study included the small number of participants recruited and that they were subjected to only one induction cycle, our results can be integrated in larger systemic reviews and meta-analysis. Also, we did not study the possible side effects from using ISMN in our study.

\section{CONCLUSION}

We concluded that ISMN improved the follicular number, endometrial growth and cervical mucous. Therefore, its use with CC should be tailored according to the needs of each patient to overcome the unwanted side effects of $\mathrm{CC}$ and achieve higher pregnancy rates. But it should be used cautiously as in our study it did not significantly increase the pregnancy rate.

\section{CONFLICT OF INTERESTS}

There are no conflicts of interest.

\section{REFERENCES}

1. Delcour C, Robin G, Young J, Dewailly D. PCOS and Hyperprolactinemia: what do we know in 2019?. Clin Med Insights Reprod Health. 2019; 13:1179558119871921.

2. Lizneva D, Suturina L, Walker W, Brakta S, Gavrilova-Jordan L, Azziz R. Criteria, prevalence, and phenotypes of polycystic ovary syndrome. Fertil Steril. 2016;106:6-15.

3. Brown J, Farquhar C. Clomiphene and other antioestrogens for ovulation induction in polycystic ovarian syndrome. Cochrane Database Syst Rev. 2016;12:CD002249.

4. Legro RS, BarnhartHX, SchlaffWD, etal.Clomiphene, metformin, or both for infertility in the polycystic ovary syndrome. N Engl J Med. 2007;356:551-566.

5. Homburg R. Clomiphene citrate--end of an era? A mini-review. Hum Reprod. 2005;20:2043-2051.

6. Azzam ME; Mohamed MM; Taha MO; Abozeid I. The effect of Laparoscopic Ovarian Drilling on AntiMüllerian Hormone, LH/FSH ratio and inhibin B. EBWHJ. 2020;10:37-45.

7. Moncada S, Palmer RM, Higgs EA. Nitric oxide: physiology, pathophysiology, and pharmacology. Pharmacol Rev. 1991;43:109-142.

8. Tschugguel W, Schneeberger C, Unfried G, et al. Die Rolle des Stickstoffmonoxids bei der Reproduktion [The role of nitric oxide in reproduction]. Gynakol Geburtshilfliche Rundsch. 1998;38:44-46.

9. Mörlin B, Hammarström M. Nitric oxide increases endocervical secretion at the ovulatory phase in the female. Acta Obstet Gynecol Scand. 2005;84:883-886.

10. The Rotterdam ESHRE/ASRM-sponsored PCOS consensus workshop group. Revised 2003 consensus on diagnostic criteria and long-term health risks related to polycystic ovary syndrome (PCOS) Hum Reprod. 2004;19:41-47.

11. Insler V, Melmed H, Eichenbrenner I, Serr D, Lunfeld B. The cervical score. Obstet Gynaecol. 1972;32:620625 .

12. Bassil S, Wyns C, Toussaint-Demylle D, Nisolle M, Gordts S, Donnez J. The relationship between ovarian vascularity and the duration of stimulation in invitro fertilization. Hum Reprod. 1997;12:1240-1245. 
13. Franks S, Stark J, Hardy K. Follicle dynamics and anovulation in polycystic ovary syndrome. Hum Reprod Update. 2008;14:367-378.

14. Adashi EY, Resnick CE, Hurwitz A, et al. Insulin-like growth factors: the ovarian connection. Hum Reprod. 1991;6:1213-1219.

15. Van Buren GA, Yang DS, Clark KE. Estrogen-induced uterine vasodilatation is antagonized by L-nitroarginine methyl ester, an inhibitor of nitric oxide synthesis. Am J Obstet Gynecol 1992;167:828-33.

16. Abdel Razik M, El-Berry S, Mostafa A. The effects of nitric oxide donors on uterine artery and subendometrial blood flow in patients with unexplained recurrent abortion. J Reprod Infertil 2014;15:142-6.

17. El-Berry S, Abdel Razik M. Nitric oxide donors increases pregnancy rate in clomiphene citrate treated polycystic ovary infertile patients. Middle East Fertility Society Journal 2010;2:106-109.

18. Mahran A, Abdelmeged A, Shawki H, Moheyelden A, Ahmed AM. Nitric oxide donors improve the ovulation and pregnancy rates in anovulatory women with polycystic ovary syndrome treated with clomiphene citrate: A RCT. Int J Reprod Biomed (Yazd). 2016;14(1):9-14.

19. Abdel Razik M, El-Berry S, El-Nezamy A, Saad A, Abdel Wahab A. Nitric oxide donors increase the pregnancy rate in patients with unexplained infertility undergoing clomiphene citrate stimulation and intrauterine insemination: a randomized controlled pilot study. Gynecol Endocrinol. 2017;33:199-202.

20. Ajam KA, Farzadi L, Nouri M, Sadagheani MM. The effect of nitric oxide with minimal stimulation on patients with polycystic ovarian syndrome. Int $\mathrm{J}$ Women's Health Reproductive Sci. 2014;2:121-131. 